\title{
Photocatalytic green synthesis of piperonal in aqueous $\mathrm{TiO}_{2}$ suspension
}

\author{
Marianna Bellardita ${ }^{a}$, Vittorio Loddo ${ }^{a}$, Giovanni Palmisano ${ }^{a}, \mathrm{~b}$, Ivana Pibiri ${ }^{\mathrm{c}}$, \\ Leonardo Palmisano ${ }^{\mathrm{a}, *}$, Vincenzo Augugliaro ${ }^{\mathrm{a}}$ \\ a "Schiavello-Grillone" Photocatalysis Group, Dipartimento di Energia, Ingegneria dell'informazione, e modelli Matematici (DEIM), Università degli Studi di \\ Palermo, Viale delle Scienze, Ed. 6, Palermo, Italy \\ ${ }^{\mathrm{b}}$ Istituto per i Processi Chimico Fisici, CNR, Messina, Italy \\ c Dipartimento di Scienze e Tecnologie Biologiche, Chimiche e Farmaceutiche, Università degli Studi di Palermo, Viale delle Scienze, Palermo, Italy
}

\section{A R T I C L E I N F O}

\section{Article history:}

Received 27 June 2013

Received in revised form 26 July 2013

Accepted 30 July 2013

Available online $\mathrm{xxx}$

\section{Keywords:}

Photocatalysis

Green synthesis

Piperonal

$\mathrm{TiO}_{2}$ suspension

\begin{abstract}
A B S T R A C T
Piperonal (heliotropine or 3,4-methylenedioxybenzaldehyde) has been synthesized by oxidizing piperonyl alcohol in aqueous UV-irradiated $\mathrm{TiO}_{2}$ suspensions. This compound was identified by GC-MS chromatography, ${ }^{1} \mathrm{H}$ NMR and melting point determination. The other products of the photoprocess were 1,3-bis(3,4-(methylenedioxy)benzyl) ether (found in traces) and $\mathrm{CO}_{2}$, derived from the parallel pathway of photo-mineralization. Commercial and home-prepared $\mathrm{TiO}_{2}$ samples have been tested and the best selectivity (ca. 35\%) was obtained by using the home-prepared ones. The reported green process allows to obtain an added value product (piperonal), upon partial oxidation of a cheap reagent.
\end{abstract}

(c) 2013 Elsevier B.V. All rights reserved.

\section{Introduction}

The photocatalytic and photoelectrocatalytic methods have been recently used for performing organic reactions in water in order to obtain aldehydes by partial oxidation of both aliphatic and aromatic alcohols [1-4]. Naked and doped $\mathrm{TiO}_{2}$ have been mainly used as the photocatalysts and home-prepared weakly oxidant samples generally showed much better performances than commercial $\mathrm{TiO}_{2}$ samples in terms of process selectivity [5,6]. The absence of harmful stoichiometric oxidants and/or heavy-metal polluting catalysts together with the use of $\mathrm{H}_{2} \mathrm{O}$ as the solvent allows to refer to these processes as green. These methods could be alternative to traditional ones after investigating optimal arrangements of the process. A recent development in this field resulted in a photoreactor integrated by a pervaporation membrane separation unit able to prevent overoxidation of the product of interest [7].

Piperonal is a molecule widely used as basis for the heliotropetype perfumes and for cosmetic preparations, being also applied as an intermediate for agrochemical and pharmaceutical products. It has been moreover demonstrated that piperonal has

\footnotetext{
* Corresponding author. +3909123863746.

E-mail addresses: marianna.bellardita@unipa.it (M. Bellardita), leonardo.palmisano@unipa.it (L. Palmisano).
}

powerful aroma-therapeutic qualities able to elevate mood and to impart a general well-being [8-11]. Piperonal was traditionally produced by isomerization of safrole $[12,13]$, isolated from the essential oil of Ocotea trees and Cymbarum Pretiosa, and its subsequent oxidation [14,15]. For many years this was the only way for producing piperonal. The present industrial processes use 1,2-methylenedioxybenzene [16] or 3.4-dihydroxybenzaldehyde $[17,18]$ as reagents or they exploit the oxidation of the piperonyl alcohol $[19,20]$. Catalytic synthesis of piperonal involves the chloromethylation of methylenedioxybenzene (1,3-benzodioxole) to piperonylchloride (3,4-methylenedioxybenzyl chloride), followed by reaction with the sodium salt of 2-nitropropane in an alcoholic solvent [21]. Catalytic oxidation of organic halides to aldehydes and ketones with $\mathrm{H}_{5} \mathrm{IO}_{6}$ in ionic liquid has been also developed [22]. Another synthetic route is the vanillin demethylation with pyridine and $\mathrm{AlCl}_{3}$ [23]. These routes make all use of harmful reagents or environmentally unsafe heavy metals. The natural resources, moreover, are becoming expensive, less available and non-renewable, thus increasing the demand for alternative, ecologically and economically sustainable synthetic procedures.

Synthesis of piperonal and its separation and characterization is here presented for the first time by applying the photocatalytic method. Piperonyl alcohol (ca. 500 times less expensive than piperonal) was used as the starting reagent. Aqueous suspensions of commercial (Merck or Degussa P25) and home prepared (ex $\mathrm{TiCl}_{4}$ or ex $\left.\mathrm{TiOSO}_{4}\right) \mathrm{TiO}_{2}$ were irradiated by a $125 \mathrm{~W}$ medium pressure 
$\mathrm{Hg}$ lamp in an annular batch reactor, and no detectable by-product was detected in water but $\mathrm{CO}_{2}$ and trace amounts of 1,3-bis(3,4(methylenedioxy)benzyl) ether.

\section{Experimental}

\subsection{Materials}

Titanium tetrachloride (Fluka 98\%) and Titanium(IV) oxysulfate (Sigma-Aldrich $\geq 29 \% \mathrm{Ti}\left(\right.$ as $\left.\mathrm{TiO}_{2}\right)$ ) were used as precursors without any further purification. Commercial anatase (Merck) and Degussa P25 $\mathrm{TiO}_{2}$ samples were also used as provided for the sake of comparison with the home prepared samples.

\subsection{Samples preparation}

Home prepared samples were obtained from an aqueous $\mathrm{TiO}_{2}$ sol synthesized by controlled hydrolysis of $\mathrm{TiCl}_{4}$, later being either boiled [24] (HP0.5) or kept at room temperature (HPRT) [25]. Another sample was obtained by heating at $373 \mathrm{~K}$ an aqueous $\mathrm{TiO}_{2}$ sol obtained from $\mathrm{TiOSO}_{4}$ (HP873S) [26].

HP0.5: Titanium tetrachloride (Fluka 98\%) was used as the precursor without any further purification. It was slowly added to distilled water (molar ratio $\mathrm{Ti} / \mathrm{H}_{2} \mathrm{O}$ 1:60; volume ratio 1:10) at room temperature. After ca. $12 \mathrm{~h}$ of continuous stirring at room temperature, a clear solution was obtained. The clear solution was boiled for $0.5 \mathrm{~h}$ under agitation. This treatment produced a milky white $\mathrm{TiO}_{2}$ dispersion; the solid was dried under vacuum at $323 \mathrm{~K}$. HPRT: $20 \mathrm{~mL}$ of $\mathrm{TiCl}_{4}$ (>97\%, Fluka) were added to $1000 \mathrm{~mL}$ water contained in a volumetric flask; the addition was carried out very slowly without agitation in order to avoid the warming of the solution as the $\mathrm{TiCl}_{4}$ hydrolysis is a highly exothermic reaction. At the end of the addition, the resulting solution was mixed for 2 min by a magnetic stirrer and then the flask was sealed and maintained at room temperature (ca. $298 \mathrm{~K}$ ) for 6 days. Just after ca. $12 \mathrm{~h}$ aging, the sol became a transparent solution and then, after waiting a few days, the precipitation process started. The solid powder precipitated at the end of the whole treatment was dialyzed (Dialysis Tubing Membrane with MW 12,400 cut-off pores) against $4 \mathrm{dm}^{3}$ of water replaced every day, at room temperature. The final $\mathrm{pH}$ of the permeate was 5 . The solid was recovered using a vacuum pump at $323 \mathrm{~K}$.

HP873S: Titanium(IV) oxysulfate (Sigma-Aldrich) was used as the precursor. $20 \mathrm{~g}$ of $\mathrm{TiOSO}_{4}$ were added to $90 \mathrm{~mL}$ of distilled water. After ca. $2 \mathrm{~h}$ of continuous stirring at room temperature, a clear solution was obtained. This solution was heated in a closed bottle and aged at $373 \mathrm{~K}$ in an oven for $48 \mathrm{~h}$. The resultant precipitate was washed by withdrawing many times the supernatant liquid and by adding pure water to restore the initial solution volume and to eliminate most of the sulfate ions. The resultant solid was recovered using a vacuum pump at $323 \mathrm{~K}$ and calcined at $873 \mathrm{~K}$ for $10 \mathrm{~h}$.

\subsection{Samples characterization}

X-ray diffractometry (XRD) patterns of the powders were recorded by a Philips diffractometer (operating at a voltage of $40 \mathrm{kV}$ and a current of $30 \mathrm{~mA}$ ) using the $\mathrm{Cu} \mathrm{K} \alpha$ radiation and a $2 \theta$ scan rate of $1.28^{\circ} / \mathrm{min}$. The crystalline sizes of the samples were determined by using the Scherrer equation [27]. The specific surface areas (SSA) of the powders were determined in a Flow Sorb 2300 apparatus (Micromeritics) by using the single-point BET method. The samples were degassed for $0.5 \mathrm{~h}$ at $523 \mathrm{~K}$ prior to the measurement (see Table 1).

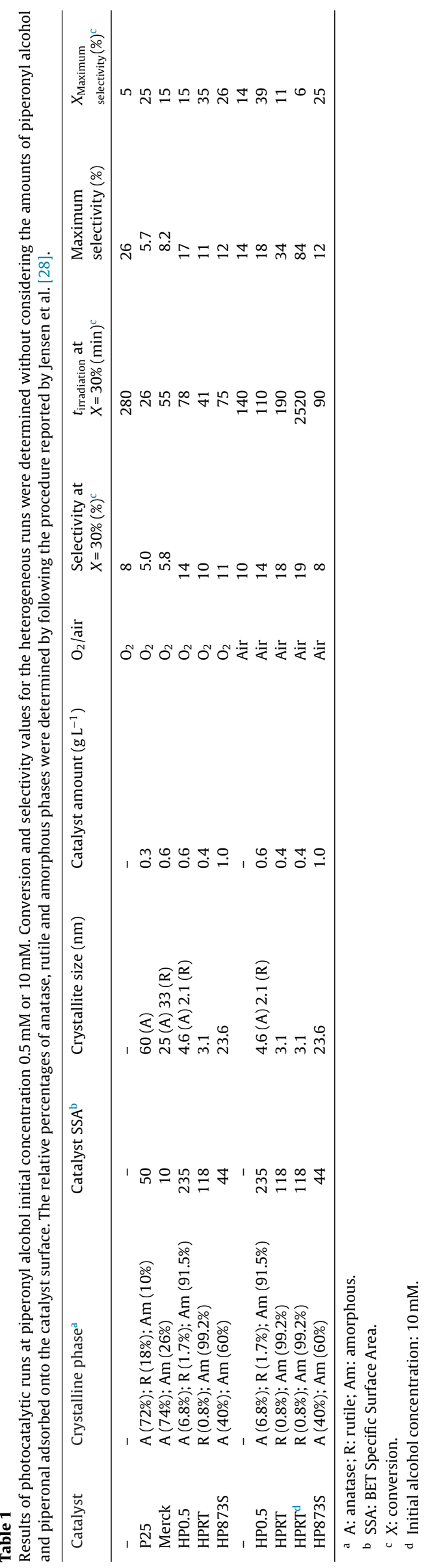




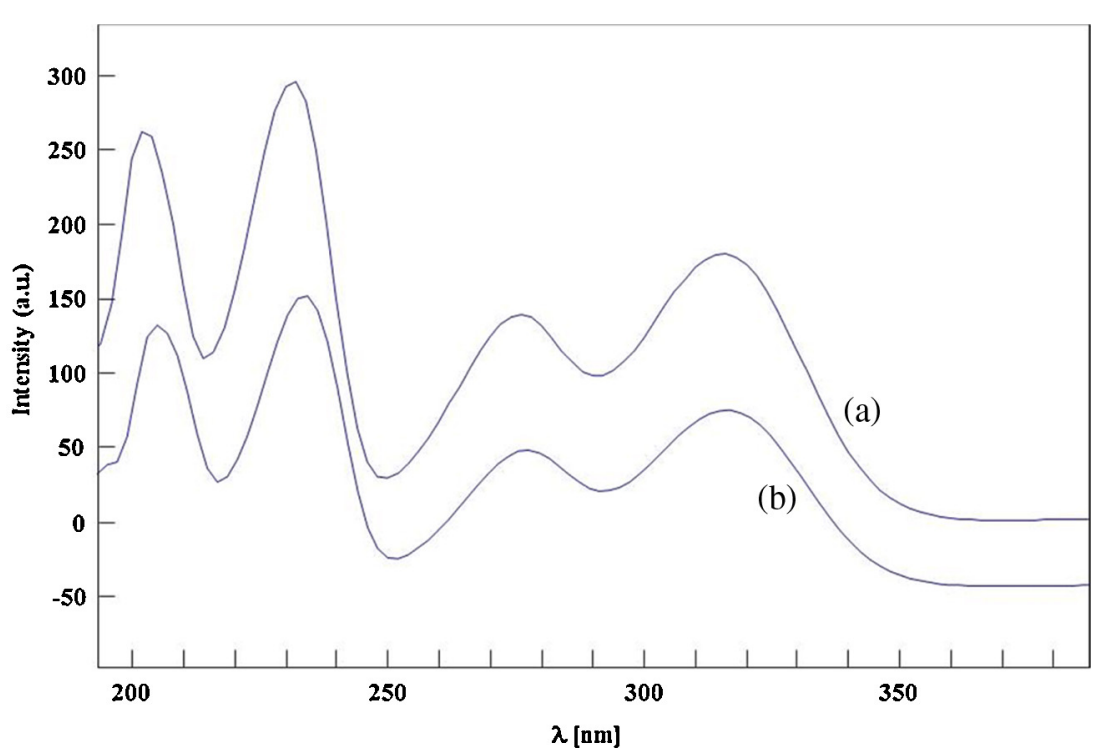

Fig. 1. Absorbance UV-vis spectra of (a) the obtained and (b) the purchased piperonal.

Total organic carbon (TOC) analyses were carried out by using a 5000A Shimadzu TOC analyzer. These analyses allowed to determine the amount of organic carbon mineralized to $\mathrm{CO}_{2}$ in the course of the reaction.

The crystallinity of all of the samples was evaluated following the procedure by Jensen et al. [28]. XRD diffractograms were recorded for mixtures of $\mathrm{TiO}_{2}$ and $\mathrm{CaF}_{2}(50 \%, \mathrm{w} / \mathrm{w})$ and the areas of the $100 \%$ peaks of anatase (lllll 01 , rutile $\left(\begin{array}{lll}1 & 1 & 0\end{array}\right)$ and $\mathrm{CaF}_{2}\left(\begin{array}{lll}2 & 2 & 0\end{array}\right)$ were determined. By comparing the ratio between the areas of $(1$ 01 ) and (2 20 ) peaks or of ( $\left.\begin{array}{lll}1 & 1 & 0\end{array}\right)$ and (2 20 ) peaks to the ratios obtained by using the pure phases ( 1.25 for anatase and 0.90 for rutile), the amount of crystalline and amorphous phases present in the samples was determined.

\subsection{Experimental setup and HPLC analysis}

A Pyrex batch photoreactor of cylindrical shape, containing $0.5 \mathrm{~L}$ of aqueous suspension, was used for performing the reactivity experiments. The photoreactor was provided with ports in its upper section for the inlet and outlet of gases and for sampling. A magnetic stirrer guaranteed a satisfactory suspension of the photocatalyst and the uniformity of the reacting mixture. A $125 \mathrm{~W}$ medium pressure Hg lamp (Helios Italquartz) was axially immersed within the photoreactor and it was cooled by water circulating through a Pyrex thimble; the temperature of the suspension was about $300 \mathrm{~K}$. The radiation energy impinging on the suspension had an average value of $10 \mathrm{~mW} \mathrm{~cm}^{-2}$; it was measured by using a radiometer UVX Digital, at $\lambda=360 \mathrm{~nm}$.

Different amounts of the catalysts (ranging from 0.3 to $1.0 \mathrm{~g} \mathrm{~L}^{-1}$ ) were used for the reactivity runs; they were determined in order to have the same photon flux absorbed by the suspension for each catalyst. That amount was determined by slowly adding the powder and by measuring the photon flux transmitted by the suspension; when the transmitted radiation was less than $10 \%$ with respect to the incident one, catalyst addition was stopped. The starting concentration of piperonyl alcohol was generally $0.5 \mathrm{mM}$. Experiments were also carried out starting from initial concentrations of piperonyl alcohol equal to 5 or $10 \mathrm{mM}$. The initial $\mathrm{pH}$ of the suspensions was the natural one (ca. 5). The suspensions were saturated by bubbling $\mathrm{O}_{2}$ or air at atmospheric pressure for $0.5 \mathrm{~h}$ in the dark and throughout all the runs. Some runs were performed by irradiating the piperonylic alcohol solution to investigate the contribution of the homogeneous reaction. Blank tests carried out under dark indicated that no transformation of the alcohol occurred.

Samples for analyses were withdrawn at fixed intervals of time; the catalyst was immediately separated from the aqueous solution by filtering through $0.2 \mu \mathrm{m}$ Millex Millipore filters.

Piperonyl alcohol and piperonal were analyzed by means of a HPLC Beckman Coulter (System Gold 126 Solvent Module and 168 Diode Array Detector), equipped with $3 \mu$ Dionex Acclaim PA2. The eluent consisted of a mixture of acetonitrile and $1 \mathrm{mM}$ trifluoroacetic acid aqueous solution (20:80 v:v). The flow rate was $0.8 \mathrm{~mL} / \mathrm{min}$ and the identification was carried out by comparing retention times and UV-vis absorbance spectra of unknown peaks with those of authentic standard samples (purchased by Sigma-Aldrich and LGC Standards S.r.L.). Fig. 1 reports the absorbance UV-vis spectra of the obtained and the purchased piperonal. All the maxima and minima and also the general trend finely match each other. It is worth noting that the HPLC chromatograms did not reveal any significant peak but those attributed to piperonyl alcohol and piperonal.

\subsection{GC-MS, NMR and experimental details}

Melting points were determined on a Reichart-Thermovar hotstage apparatus and are uncorrected. ${ }^{1} \mathrm{H}$ NMR spectra $(250 \mathrm{MHz})$ where taken on a Bruker $250 \mathrm{E}$ spectrometer; ${ }^{13} \mathrm{C}$ NMR spectrum (75.5 MHz) was taken on a Bruker 300 Avance spectrometer. Flash chromatography was performed using silica gel (200-400 mesh) and mixtures of EtOAc and light petroleum (fraction boiling in the range $40-60^{\circ} \mathrm{C}$ ) in various ratios.

GC-MS allowed a further identification of piperonal. A Thermo Fisher ITQ900 instrument was used, equipped with a Thermo Scientific TG-WAXMS A $30 \mathrm{~m} \times 0.25 \mathrm{~mm} \times 0.25 \mu \mathrm{m}$ column. A temperature ramp was applied as follows: $150^{\circ} \mathrm{C}$ for $4 \mathrm{~min}, 1.5^{\circ} \mathrm{C} / \mathrm{min}$ up to $250^{\circ} \mathrm{C}$ and $40 \mathrm{~min}$ at $250^{\circ} \mathrm{C}$. Retention time of piperonal was $18.54 \mathrm{~min}$. Fig. 2 shows the obtained spectra coming from the obtained and the purchased sample. It can be seen that parent peak and fragmentation peeks finely match.

The piperonal separated by a chromatography procedure described in Section 3 was crystallized by hexane and its purity was checked by melting point determination, ${ }^{1} \mathrm{H}$ NMR, and GC-MS analysis by comparison as for retention time and mass spectrum 

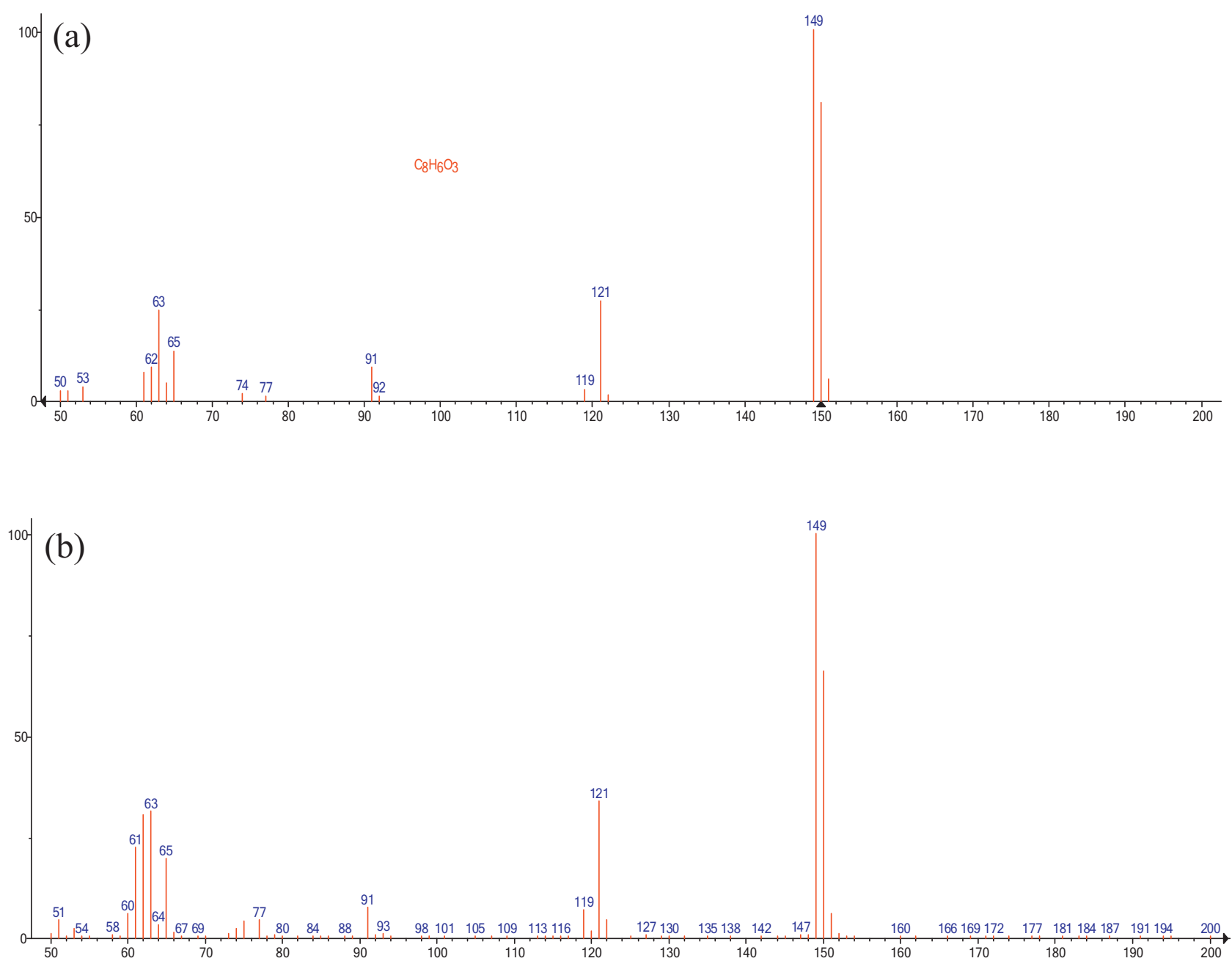

Fig. 2. GC-MS fragmentation spectra of (a) the obtained and (b) the purchased piperonal.
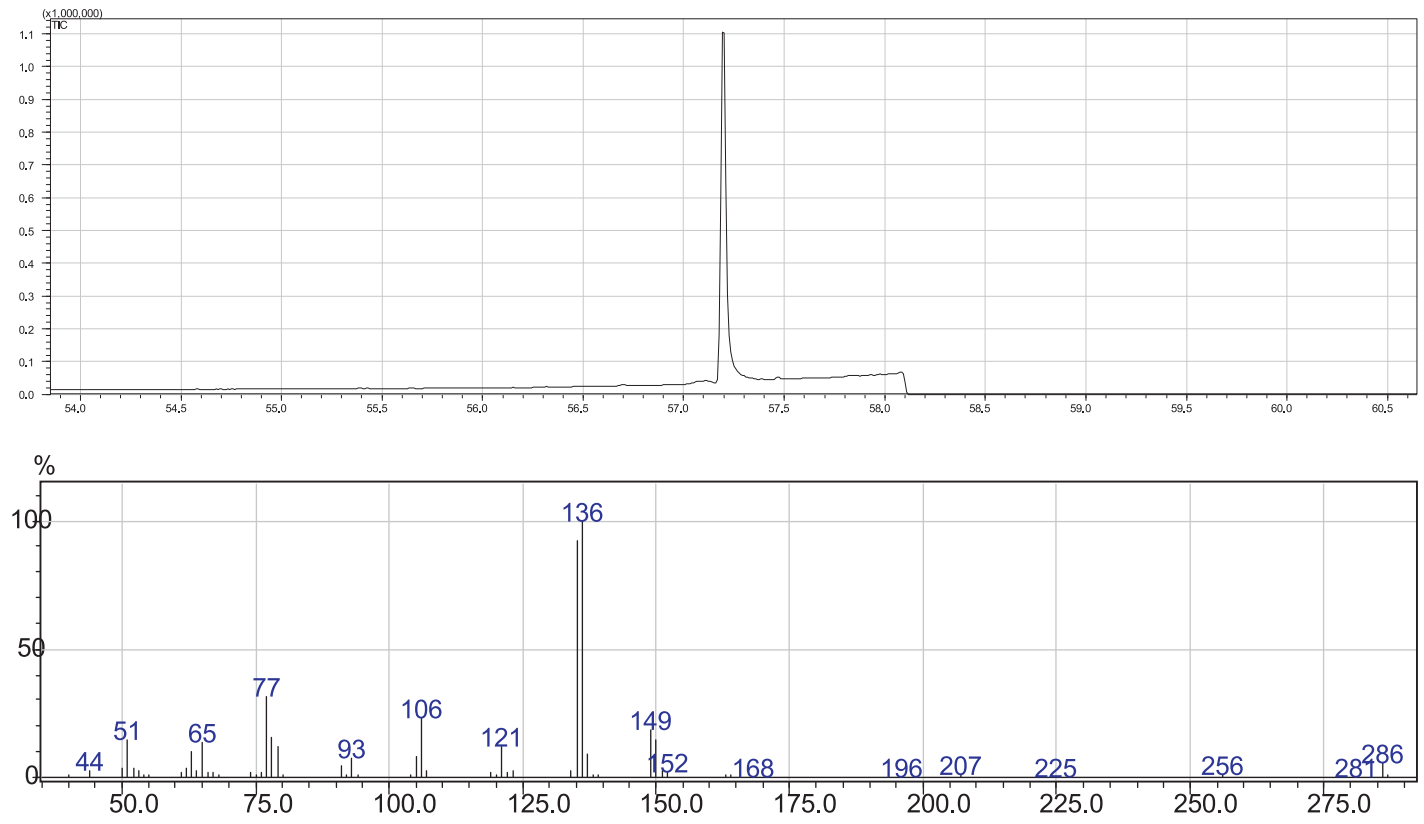

Fig. 3. GC-MS chromatogram and fragmentations relative to the 1,3-bis(3,4-(methylenedioxy)benzyl) ether. 
(a)

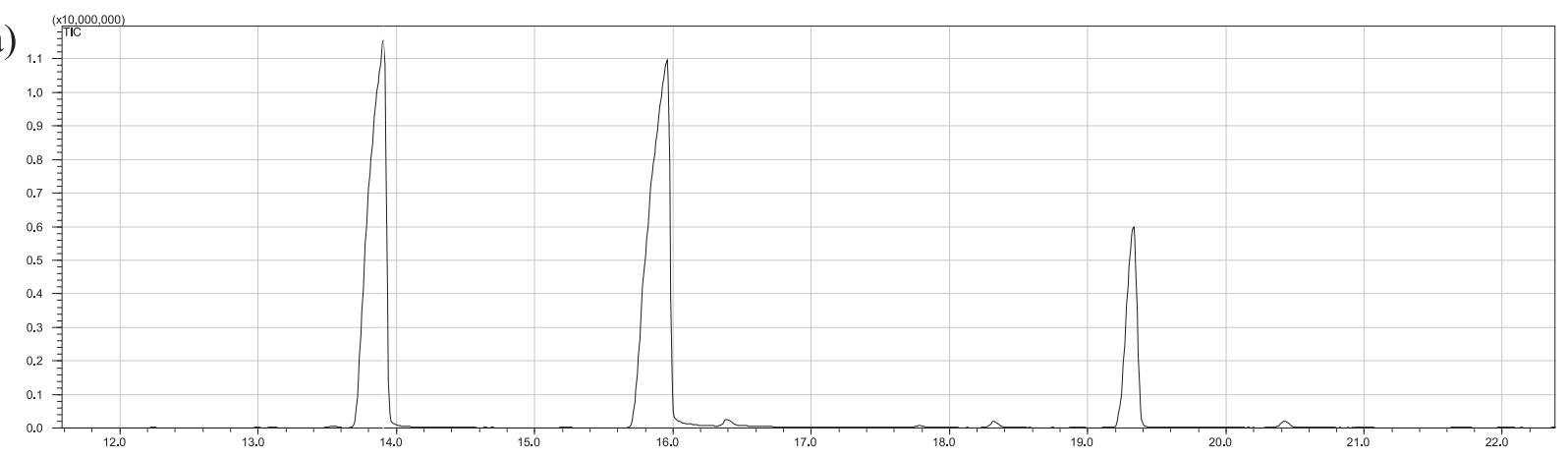

(b)

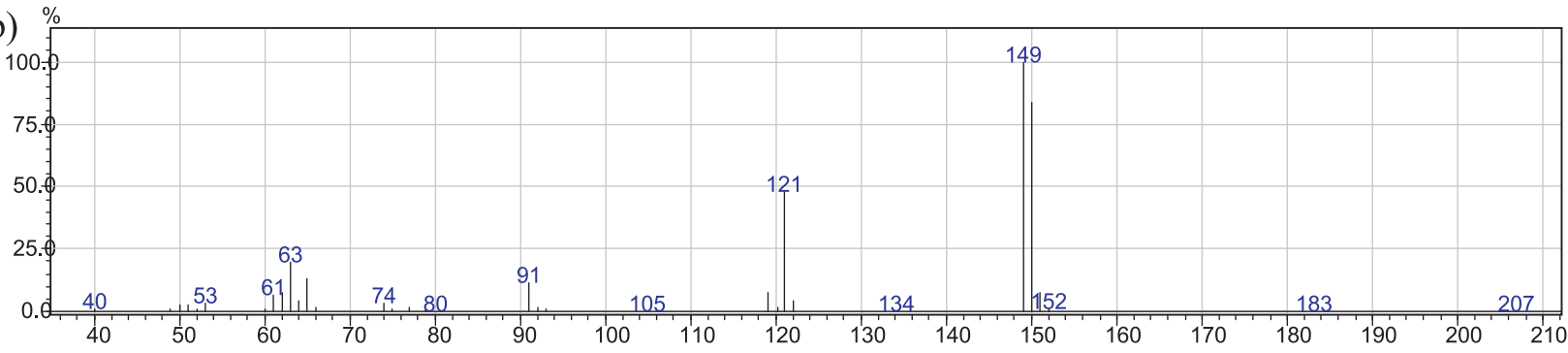

(c)

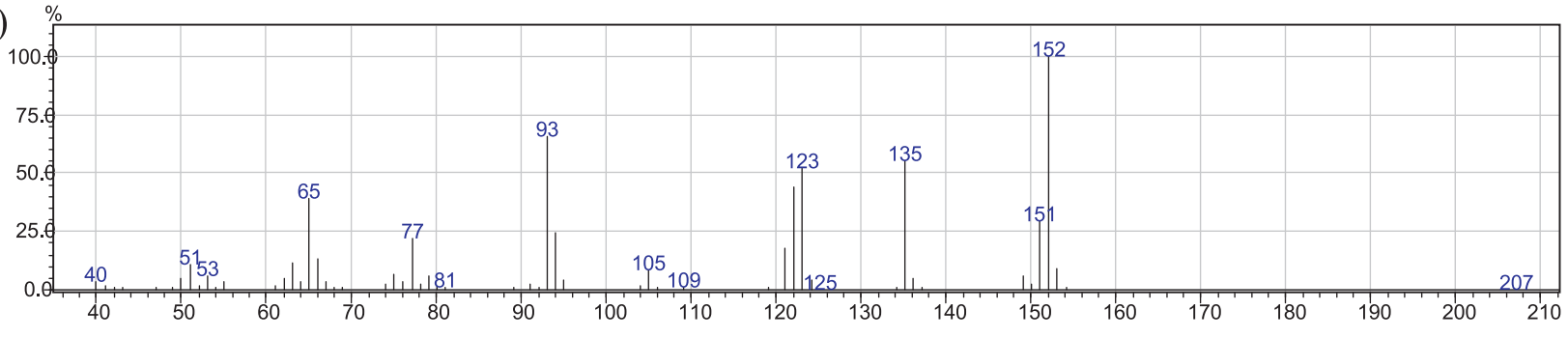

(d)

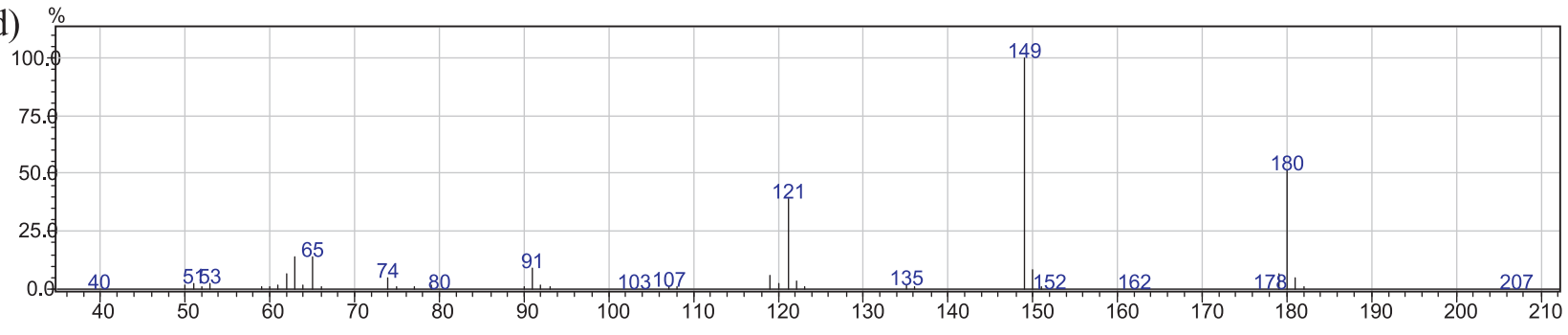

Fig. 4. GC-MS chromatogram (a) and fragmentation spectra relative to the three observed peaks of piperonal (b), piperonyl alcohol (c), piperonylic acid methyl ester (d).

with a standard sample. Piperonal: white solid, m.p. $35-37^{\circ} \mathrm{C}$ (from hexane); ${ }^{1} \mathrm{H}$ NMR $\left(250 \mathrm{MHz}, \mathrm{CDCl}_{3}\right) \delta 9.82$ (s, $\left.1 \mathrm{H}, \mathrm{CHO}\right), 7.44$ (dd, $1 H, J=8$ and $2 \mathrm{~Hz}, \mathrm{Ar}$ ), 7.35 (d, $1 H, J=2 \mathrm{~Hz}, \mathrm{Ar}), 6.95(\mathrm{~d}, 1 \mathrm{H}, J=8 \mathrm{~Hz}$, $\mathrm{Ar}), 6.09\left(\mathrm{~s}, 2 \mathrm{H}, \mathrm{OCH}_{2} \mathrm{O}\right)$; GC-MS $\left(\mathrm{m} / \mathrm{z}\right.$, relative intensity): $150\left(\mathrm{M}^{+}\right.$, 90), 149 (100), 121 (48), 91 (12), 63 (25).

For some experiments 1,3-bis(3,4-(methylenedioxy)benzyl ether synthesized and identified by NMR and GC-MS measurements was used as it was not possible to purchase a standard sample.

The 1,3-bis(3,4-(methylenedioxy)benzyl) ether has been synthesized by a one-pot procedure, by dissolving the piperonyl alcohol $(250 \mathrm{mg}, 1.64 \mathrm{mmol})$ in dry pyridine $(5 \mathrm{~mL})$, then methanesulfonyl chloride ( $63 \mu \mathrm{L}, 0.82 \mathrm{mmol}$ ) was added and the reaction was refluxed for $4 \mathrm{~h}$. The reaction mixture was worked-up with water, then extracted with diethyl ether three times. Column chromatography with petroleum/ethyl acetate 5:1 allowed to obtain the 1,3bis(3,4-(methylenedioxy)benzyl) ether (102 mg, 43\% yield). The identity of the synthesized 1,3-bis(3,4-(methylenedioxy)benzyl) ether was confirmed by ${ }^{1} \mathrm{H},{ }^{13} \mathrm{C}$ NMR, and MS spectroscopy and it exactly matches what reported in the literature [29].

${ }^{1} \mathrm{H}$ NMR $\left(250 \mathrm{MHz}, \mathrm{CDCl}_{3}\right) \delta 4.43\left(\mathrm{~s}, 4 \mathrm{H}, \mathrm{CH}_{2} \mathrm{O}\right), 5.96(\mathrm{~s}, 4 \mathrm{H}$, $\left.\mathrm{OCH}_{2} \mathrm{O}\right), 6.80$ (s, $\left.4 \mathrm{H}, \mathrm{Ar}\right), 6.88(\mathrm{~s}, 2 \mathrm{H}, \mathrm{Ar}) ;{ }^{13} \mathrm{CNMR}\left(75.5 \mathrm{MHz}, \mathrm{CDCl}_{3}\right)$ $\delta$ 71.69, 100.97, 108.07, 108.57, 121.43, 132.09, 147.12, 147.77; GC-MS ( $m / z$, relative intensity): $286\left(\mathrm{M}^{+}, 10\right), 149(20), 136(100)$, 121 (10), 106 (25), 77 (30), 63 (12), 51(15).

GC-MS analysis reported in Figs. 3 and 4 were performed on a Shimadzu GC-MS QP2010 Plus. Fig. 3 shows the GC-MS chromatogram and the relative fragmentations for the isolated 1,3bis(3,4-(methylenedioxy)benzyl) ether. Fig. 4 shows the GC-MS chromatogram and the relative fragmentations for the three observed peaks of the homogeneous run carried out starting with equimolar amounts of alcohol and aldehyde, and after derivatization by addition of freshly prepared diazomethane ethereal solution: the chromatogram showed the presence of three peaks, 


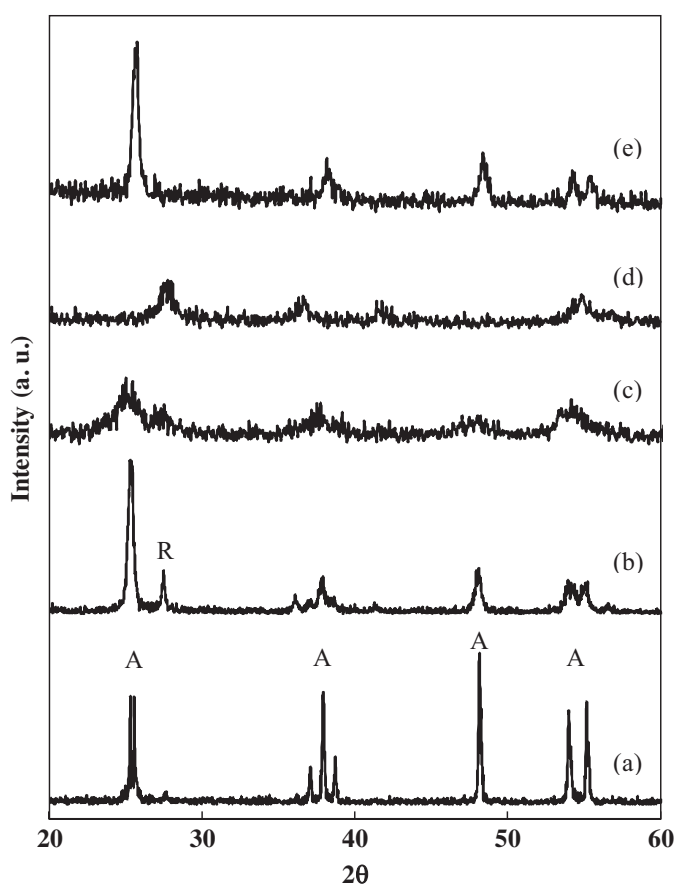

Fig. 5. XRD patterns of the samples: Merck (a), P25 (b), HP0.5 (c), HPRT (d), HP873S (e).

relative to piperonal, piperonyl alcohol and piperonylic acid methyl ester respectively.

\section{Results and discussion}

Fig. 5 reports the X-ray diffractograms of all of the samples used in this work. The various peaks corresponding to $\mathrm{TiO}_{2}$ anatase (A) and rutile $(\mathrm{R})$ phases have been identified. Commercial powders are more crystalline than those home prepared. The worst crystallized samples are HP0.5 and HPRT, with the smallest crystallite size because both catalysts were not subjected to a strong thermal treatment. HP05 is characterized by the presence of anatase as the main phase whereas HPRT is constituted only by rutile. Among the home made catalysts, HP873S, consisting of pure anatase $\mathrm{TiO}_{2}$, resulted the most crystalline.

The specific surface areas of the home prepared samples obtained from $\mathrm{TiCl}_{4}$ are higher than those of the commercial samples while the crystalline sizes are smaller because of the low preparation temperature (see Table 1 ).

Table 1 reports the results obtained by performing homogeneous and heterogeneous runs in the presence of irradiation and by bubbling $\mathrm{O}_{2}$ or air. In the dark no reactivity was observed both in homogeneous and heterogeneous systems. In the absence of $\mathrm{TiO}_{2}$ photo-oxidation of piperonyl alcohol to piperonal was observed, as

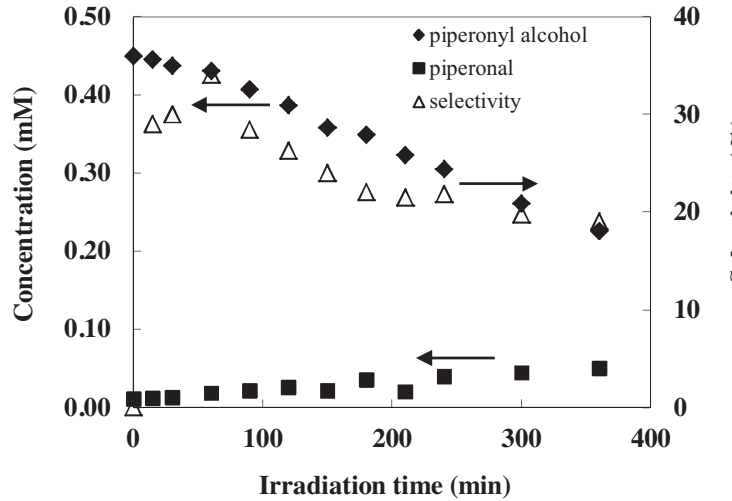

Fig. 6. Experimental values of piperonyl alcohol ( $)$ and piperonal ( $\boldsymbol{\square})$ concentrations and selectivity to piperonal $(\triangle)$ for a run carried out in the presence of HPRT and air.

the alcohol absorbs in the near UV region, where the used lamp has its main emission peak. In homogeneous system the conversion was faster in the presence of air with respect to pure $\mathrm{O}_{2}$, probably due to a quenching effect of the latter on some excited intermediate species; the selectivity to piperonal was around $8-10 \%$ at $30 \%$ alcohol conversion.

In the presence of a photocatalyst, whose amount was chosen in order to absorb ca. $90 \%$ of the emitted light, the reaction rate and the selectivity increased with respect to the homogenous cases; all their values are reported in Table 1 . The maximum selectivity values of Table 1 refer to very low alcohol conversions. Selectivity, starting from low alcohol concentration $(0.5 \mathrm{mM})$, reached a best $18 \%$ (at $30 \%$ piperonyl alcohol conversion) and a maximum value of $34 \%$ in the presence of air, by using HPRT. Oxygen improved reactivity with a detrimental effect on selectivity. The selectivity increased starting from higher substrate concentrations, and a maximum of $84 \%$ after about $42 \mathrm{~h}$ of irradiation at $6 \%$ of alcohol conversion.

All the home-prepared catalysts, which are less crystalline and more hydroxylated than Degussa P25 and Merck $\mathrm{TiO}_{2}$ [26], showed higher selectivity. Conversely the fastest reaction took place in the presence of Degussa P25. Merck performance in term of selectivity was similar to P25, but reaction rate was slower. This behavior is in accord with previous results obtained for selective oxidation of other alcohols to the corresponding aldehyde [2,24,25].

It is well known that the oxidation of aromatic species occurs through two main pathways, i.e. the direct mineralization (through a series of adsorbed intermediates which do not desorb into the bulk of solution) and the partial oxidation. As showed in Scheme 1, other products formed in addition to piperonal were $\mathrm{CO}_{2}$ and 1,3bis(3,4-(methylenedioxy)benzyl) ether; traces of this compound were found only starting from the highest concentration of alcohol solutions.

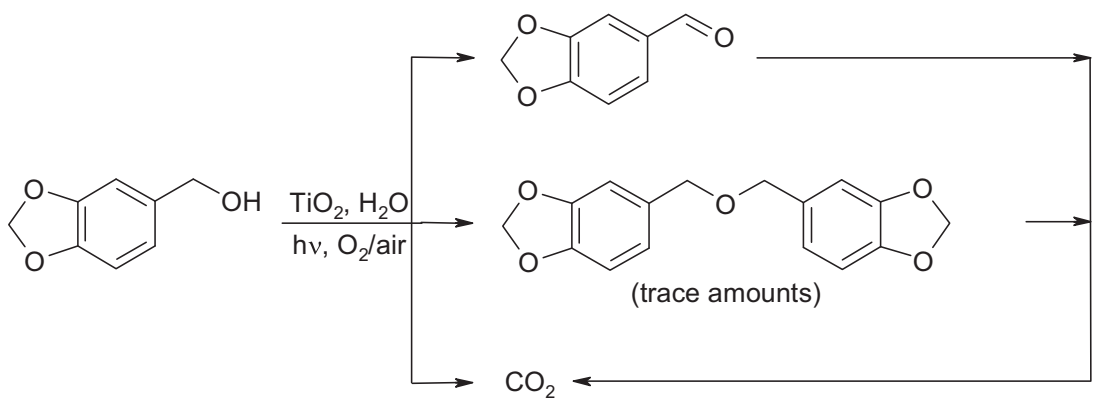

Scheme 1. Main reaction pathways. 
Fig. 6 shows a typical reaction trend: in the presence of HPRT sample piperonyl alcohol and piperonal concentrations slowly declines and raises, respectively, while piperonal selectivity initially increases, reaches a maximum and then decreases. A similar trend was observed in the presence of the others catalysts. TOC analyses indicated that the organic species present in the solution during the experiments were virtually only unreacted piperonyl alcohol and piperonal.

Photocatalytic runs starting from a piperonyl alcohol concentrations of $5 \mathrm{mM}$ or $10 \mathrm{mM}$ were performed by using HPRT; these runs lasted more than $60 \mathrm{~h}$ in order to isolate and to characterize higher amounts of the products. No significant deactivation of the photocatalysts was observed also for these long lasting experiments. At the end of the run the final aqueous suspension was treated three times with diethyl ether and subjected to ultrasound treatment to extract both the products and the unreacted alcohol. The organic layers were dried by $\mathrm{Na}_{2} \mathrm{SO}_{4}$, evaporated and separated in its components according to the chromatographic procedure below described.

Column chromatography with petroleum and ethyl acetate in various ratios allowed to obtain piperonal $(77 \mathrm{mg}, 10 \%$ isolated yield based on the starting amount of alcohol; 20\% yield, based on the converted amount of alcohol) and to recover the starting reagent piperonyl alcohol $(378 \mathrm{mg}, 49 \%$ with respect to the starting amount of $760 \mathrm{mg})$. Moreover trace amounts of 1,3-bis(3,4(methylenedioxy)benzyl) ether have been isolated ( $4 \mathrm{mg}, 0.6 \%$ isolated yield based on the starting amount of alcohol). Its identity has been determined by comparison of the GC-MS peaks and fragmentations and NMR analysis with a sample obtained by synthesis, and the two samples finely match. Notably about $40 \%$ of the starting reagent was lost during the photocatalytic process, due to the mineralization pathway to $\mathrm{CO}_{2}$ and to the isolation procedures.

Notably only for the runs carried out with high initial alcohol concentrations the surface of catalyst appeared slightly ochre colored. The coloration is due to traces of piperonal, piperonyl alcohol and ether adsorbed onto the catalyst surface, as confirmed by chromatographic extraction.

Runs starting from $0.07 \mathrm{mM}$ of 1,3-bis(3,4-(methylenedioxy) benzyl ether in the presence of $0.6 \mathrm{~g} \mathrm{~L}^{-1}$ HP0.5 or HPRT showed that about $40-50 \%$ of the substrate was adsorbed in the dark after $0.5 \mathrm{~h}$ mixing. Notably the catalyst turned its color from white to yellowish. Upon irradiation of the suspension containing the HP0.5 sample degradation occurred slowly, with about 30\% decrease of ether in solution after ca. $3 \mathrm{~h}$. Piperonyl alcohol and piperonal were not formed.

An in-depth investigation was carried out in order to understand if the formed ether derived from alcohol, aldehyde or both. During homogeneous runs carried out starting from piperonyl alcohol $(0.5 \mathrm{mM})$, the formation of piperonal and traces of ether was observed. Homogeneous runs carried out starting with equimolar amounts of alcohol $(0.25 \mathrm{mM})$ and aldehyde $(0.25 \mathrm{mM})$ showed that ether did not form but piperonylic acid was noticed in significant amount. Indeed, as shown by TLC analysis, a more polar spot was detected together with the spots relative to piperonyl alcohol and piperonal. By addition of freshly prepared diazomethane ethereal solution to the reaction solution, it was possible to observe the formation of a less polar product and the disappearance of the more polar spot; the so derivatized solution was analyzed by GC-MS and the chromatogram (Fig. 4) showed the presence of three peaks, relative to piperonal, piperonyl alcohol and piperonylic acid methyl ester respectively. Finally a run starting from piperonal $(0.5 \mathrm{mM})$ showed only the formation of piperonylic acid.

Heterogeneous runs carried out by using HPRT and starting from aldehyde did not give rise to any oxidation products but $\mathrm{CO}_{2}$, probably because piperonylic acid is adsorbed and photodegraded on the catalyst surface. Aldehyde reactivity was moreover very low with respect to the alcohol, indicating that this molecule is relatively stable in the reaction medium. The above findings carry to the final conclusion that the ether is formed by coupling of two molecules of piperonyl alcohol both in homogeneous and heterogeneous runs and it is not an intermediate of piperonal.

\section{Conclusions}

Piperonal, an added value product, has been obtained by an environmentally safe process, exploiting selective photocatalytic oxidation of piperonyl alcohol, a precursor approximately 500 times less expensive, in organic-free aqueous UV-irradiated home prepared and commercial $\mathrm{TiO}_{2}$ suspensions. The best selectivity (ca. $35 \%$ starting from $0.5 \mathrm{mM}$ solution of piperonyl alcohol) was obtained by using the home-prepared samples. Trace amounts of 1,3-bis(3,4-(methylenedioxy)benzyl) ether were also produced, but only starting from high alcohol concentrations. The virtual absence of by-products makes easy the separation of piperonal from the reacting solution by a chromatographic procedure. The low cost of piperonyl alcohol with respect to piperonal and the mild experimental conditions used makes this process attractive by an application point of view, although the selectivity to piperonal is not very high as a portion of the starting reagent is lost due to its mineralization to $\mathrm{CO}_{2}$.

\section{References}

[1] M. Bonchio, M. Carraro, G. Scorrano, E. Fontananova, E. Drioli, Adv. Synth. Catal. 345 (2003) 1119-1126.

[2] S. Yurdakal, G. Palmisano, V. Loddo, V. Augugliaro, L. Palmisano, J. Am. Chem. Soc. 130 (2008) 1568-1569.

[3] A. Molinari, M. Montoncello, H. Rezala, A. Maldotti, Photochem. Photobiol. Sci. 8 (2009) 613-619.

[4] L. Özcan, S. Yurdakal, V. Augugliaro, V. Loddo, S. Palmas, G. Palmisano, L. Palmisano, Appl. Catal. B: Environ. 132-133 (2013) 535-542.

[5] S. Yurdakal, V. Loddo, G. Palmisano, V. Augugliaro, H. Berber, L. Palmisano, Ind. Eng. Chem. Res. 49 (2010) 6699-6708.

[6] S. Yurdakal, V. Augugliaro, V. Loddo, G. Palmisano, L. Palmisano, New J. Chem. 36 (2012) 1762-1768.

[7] G. Camera-Roda, F. Santarelli, V. Augugliaro, V. Loddo, G. Palmisano, L. Palmisano, S. Yurdakal, Catal. Today 161 (2011) 209-213.

[8] H. Marquez Alvarez, D.P. Barbosa, A.T. Fricks, D.A.G. Aranda, R.H. Valdés, O.A.C. Antunes, Org. Proc. Res. Dev. 10 (2006) 941-943.

[9] F. Cortés-Salazar, E. Avella-Moreno, M.T. Cortés, M. Fidel Suárez-Herrera, J. Electroanal. Chem. 606 (2007) 1-7.

[10] J. Aukunuru, K. Eedula, V. Pasham, V. Katla, S. Reddy K, Int. J. Pharm. Sci. Nanotechnol. 2 (2009) 435-442.

[11] H.-R. Bjørsvik, L. Liguori, F. Minisci, Org. Proc. Res. Dev. 4 (2000) 534-543.

[12] A.J. Hubert, H. Reimlinger, Synthesis 97 (1967) 1325-1328.

[13] G. Salmoria, E. Dall'Oglio, C. Zucco, Synth. Commun. 27 (1997) 4335-4340.

[14] J. Grimshaw, G.C. Hua, Electrochim. Acta 39 (1994) 497-499.

[15] D. Kishore, S. Kannan, Green Chem. 4 (2002) 607-610.

[16] S. Smith, B. Michael, J. March, March's Advanced Organic Chemistry: Reactions, Mechanisms, and Structure, John Wiley, New York, 2001.

[17] S. Umemura, N., Takamitsu, T., Enomiya, H. Shiraishi, German Pat. 2,804,063 (1978).

[18] Mitsui Toatsu Chemicals, French Pat. 39,056 (1977).

[19] H. Adkins, R.M. Elofson, A.G. Rossow, C.C. Robinson, J. Am. Chem. Soc. 71 (1949) 3622-3629.

[20] K. Kaneda, T. Yamashit, T. Matsushita, J. Org. Chem. 63 (1998) 1750-1751.

[21] J. Warcup Cornforth, J.E. Hawes, British Pat. 1,538,214 (1979).

[22] Y.L. Hu, Q.F. Liu, T.T. Lu, M. Lu, Catal. Commun. 11 (2010) 923-927.

[23] R.G. Lange, J. Org. Chem. 27 (1962) 2037-2039.

[24] G. Palmisano, S. Yurdakal, V. Augugliaro, V. Loddo, L. Palmisano, Adv. Synth. Catal. 349 (2007) 964-970.

[25] S. Yurdakal, G. Palmisano, V. Loddo, O. Alagöz, V. Augugliaro, L. Palmisano, Green Chem. 11 (2009) 510-516.

[26] M. Bellardita, V. Augugliaro, V. Loddo, B. Megna, G. Palmisano, L. Palmisano, M.A. Puma, Appl. Catal. A: Gen. 441-442 (2012) 79-89.

[27] P. Scherrer, Nachr. Ges. Wiss. Göttingen, Math. Phys. Kl. 2 (1918) 98-100.

[28] H. Jensen, K.D. Joensen, J.E. Jørgensen, J.S. Pedersen, E.G. Søgaard, J. Nanopart. Res. 6 (2004) 519-526.

[29] T. Takada, M. Arisawa, M. Gyoten, R. Hamada, H. Tohma, Y. Kita, J. Org. Chem. 63 (1998) 7698-7706. 